\title{
Archéopages Archéopages
}

Archéologie et société

\section{Expérimentation de la photogrammétrie en cadre préventif : relevés de vestiges de l'agglomération antique d'Intaranum/ Entrains-sur-Nohain (58)}

\section{Stéphane Venault}

\section{CpenEdition}

Journals

Édition électronique

URL : https://journals.openedition.org/archeopages/513

DOI : 10.4000/archeopages.513

ISSN : 2269-9872

Éditeur

INRAP - Institut national de recherches archéologiques préventives

Édition imprimée

Date de publication : 1 août 2014

Pagination : 58-62

ISSN : 1622-8545

Référence électronique

Stéphane Venault, «Expérimentation de la photogrammétrie en cadre préventif : relevés de vestiges de l'agglomération antique d'Intaranum/ Entrains-sur-Nohain (58) », Archéopages [En ligne], 38 | 07/2013, mis en ligne le 01 juillet 2015, consulté le 25 janvier 2022. URL : http://

journals.openedition.org/archeopages/513; DOI : https://doi.org/10.4000/archeopages.513 


\section{Les orthophotographies obtenues par photogrammétrie dans la documentation archéologique État des lieux et perspective}

L'intérêt pour la photogrammétrie en archéologie est grandissant, et si les expérimentations se multiplient, de nombreux ajustements restent à faire pour que cet outil soit utilisé au meilleur de son efficacité. Suite aux articles parus sur la photogrammétrie - dans le numéro 34, pour approfondir globalement la réflexion des chercheurs et dans le numéro 35, comme aide à la restitution -, ceux rassemblés dans ce numéro portent sur la place des orthophotographies dans

\section{Expérimentation de la photogrammétrie en cadre préventif : relevés de vestiges de l'agglomération antique d'Intaranum/ Entrains-sur-Nohain (58)}

Stéphane Venault, Inrap, UMR 6249 «Chrono-environnement»

Intaranum était une agglomération galloromaine située en territoire éduen, au carrefour de plusieurs voies, permettant de contourner le massif du Morvan par le nord. Couvrant à son apogée une surface de 70 ha, elle fut occupée $\mathrm{du} \mathrm{I}^{\mathrm{er}}$ au IV ${ }^{\mathrm{e}}$ siècle de notre ère. Un projet de construction de maison individuelle, localisé dans la frange de l'habitat antique, a donné l'occasion d'ouvrir une surface de $1000 \mathrm{~m}^{2}$, fouillée de juillet à septembre 2013. Les investigations conduites par l'Inrap ont mis au jour trois propriétés indépendantes installées perpendiculairement à une voie qui structure toujours le parcellaire actuel. Deux principales phases d'occupation ont été mises en évidence. La première correspond à l'installation de forges de la fin du $\mathrm{I}^{\mathrm{er}}$ siècle au la documentation générale de la fouille. Un seul jeu de clichés permet en effet de produire divers documents pour divers usages tant sur le terrain que pour l'analyse des données : nuages de points denses et modèles numériques pour visualisation des vestiges ; orthophotographies géoréférencées en plan, en coupe et en élévation servant d'aide au relevé, à l'observation, au travail dans un SIG et à la présentation des données.
II $^{\mathrm{e}}$ siècle. La seconde voit, au cours du III ${ }^{\mathrm{e}}$ siècle, l'édification de bâtiments d'habitation dotés de thermes. Les installations balnéaires étaient alimentées par des puits, dont l'un a servi à ensevelir un charnier daté du haut Moyen Âge, composé de plusieurs dizaines de squelettes attribués à des individus de tous âges.

Une série de structures a été enregistrée par méthode photogrammétrique dont la mise en œuvre a été assurée par la société Captair, qui est intervenue six fois sur le site, en milieu et en fin d'opération. Le traitement de cette documentation n'ayant pas encore débuté, nous ne disposons pas encore du recul nécessaire pour estimer le véritable bénéfice que constitue son exploitation par rapport aux relevés traditionnels. Nous pouvons toutefois rendre compte des applications concrètes du procédé en cours de fouille et des implications qu'il a eues sur les méthodes de travail.

Si la direction scientifique de l'Inrap Grand-EstSud avait déjà pu assister à des démonstrations techniques sur quelques chantiers, c'est sur le site 
d'Entrains que la photogrammétrie a été véritablement mise à profit comme méthode de relevé pour produire la documentation primaire. Aussi la mise en pratique de cette technique revêtait avant tout un caractère expérimental avec tous les échecs, corrections et ajustements que cela suppose.

\section{Trois objets d'étude}

Le procédé a été utilisé pour répondre à trois objectifs principaux : le dessin pierre à pierre des murs des bâtiments, le relevé en plan des différentes couches d'ossements composant le charnier et la restitution tridimensionnelle du volume du puits.

Une première campagne de photographies aériennes a été réalisée au moyen d'une caméra aéroportée prenant des clichés en continu. L'appareil était embarqué sur un drone qui a survolé l'ensemble du chantier, tout d'abord à une altitude suffisamment élevée pour couvrir la totalité de l'emprise, puis à seulement 5-6 m de hauteur pour procéder à des prises de vue rapprochées sur des secteurs plus précis. Les photos ont été géoréférencées d'après des repères topographiques matérialisés au sol et visibles sur les clichés. Les images ont été récupérées depuis un serveur quelques jours après la phase d'acquisition. Leur résolution se révélant insuffisante pour pouvoir dessiner le contour des pierres, une seconde couverture photographique a été commandée. À l'initiative du prestataire, celle-ci a été réalisée avec un appareil photo reflex monté sur une canne tenue par un harnais. La qualité bien supérieure de l'appareil et la hauteur de prise de vue limitée à seulement $2 \mathrm{~m}$ ont permis d'obtenir des images à très haute résolution. Cependant, l'angle de vue plus restreint de chacun des clichés a nécessité un travail de mosaïquage qui a rallongé le temps de traitement informatique. La durée d'acquisition des images varie selon la méthode utilisée, allant d'une dizaine de minutes avec le drone à environ une heure avec la canne. Quoi qu'il en soit, aucun des procédés ne constitue une contrainte au bon déroulement de la fouille, si le prestataire se montre assez réactif pour intervenir au moment choisi par les archéologues.

Apparu à 4,10 m sous un bouchon argileux, l'amas d'ossements s'amoncelle sur 2,70 m de hauteur. Les nombreuses connexions osseuses conservées montrent que les corps ont été jetés entiers et qu'ils se sont décomposés à l'intérieur du puits. L'empilement des cadavres dans ce volume restreint, la verticalité du contexte taphonomique et la faible quantité de sédiments ont favorisé l'enchevêtrement des squelettes et la migration vers le bas des petits os. Malgré une prolongation de la durée de l'opération pour cause de découverte exceptionnelle, la fouille du charnier a dû s'effectuer dans un délai très contraint, d'autant que son épaisseur totale était tout à fait inconnue. Il était donc nécessaire d'utiliser un système d'enregistrement qui soit non seulement rapide, mais aussi fidèle à la réalité, afin de perdre le moins d'informations possible. L'amas d'ossements a été fouillé par passes d'épaisseur décimétrique avec un arrêt à chaque niveau d'apparition de connexions anatomiques. Chaque niveau dégagé a été relevé par photogrammétrie au moyen d'un appareil reflex manipulé manuellement. Les clichés ont été pris depuis un point de vue zénithal, l'opérateur étant suspendu par un treuil, les pieds dans le vide, au-dessus de chaque passe. Cette manière de procéder nécessitait la présence en permanence du photographe, pour un nombre d'interventions effectives limitées à deux ou trois par jour.

Une fois le puits entièrement vidé, il a été décidé de procéder au relevé photographique de l'ensemble de la paroi. L'opérateur est descendu attaché à un câble pour réaliser la couverture photographique en opérant par rotations. L'intervention, très éprouvante physiquement, a duré environ trois heures. Si une partie du volume a pu d'ores et déjà être restituée, le traitement numérique en cours rencontre des difficultés dans la reconnaissance des pixels en raison des prises de vue en lumière artificielle et de l'intensité des ombres portées. Des solutions sont à l'étude pour remédier à ce problème.

\section{Documentation fournie}

La méthode permet la production de deux types de documents à partir d'une même image : une orthophotographie géoréférencée et un nuage de points créé à partir des pixels.

Le prestataire fournit plusieurs jeux d'orthophotos à des formats différents (JPEG ou TIFF), l'intérêt essentiel étant de disposer d'un fichier géoréférencé directement exploitable par un logiciel de cartographie tel Arc Gis ou QGis. Le géoréférencement direct de l'image constitue en effet un gain de temps indéniable pour le recollement des segments de murs dessinés en pierre à pierre. Enfin, même si le travail de vectorisation des pierres n'a pas encore commencé, nous pouvons juste signaler que la lourdeur des fichiers, due à la très haute résolution de l'image, ne permet pas un affichage simultané de l'ensemble des clichés. Aussi le dessin devra-t-il être réalisé par morceau, tout d'abord avec Arc Gis pour le contour des murs, puis avec Illustrator pour le tracé pierre à pierre.

Le nuage de points offre quant à lui une vision en trois dimensions des faits archéologiques. Les documents sont exploités à l'aide d'un logiciel libre (MeshLab) qui permet d'imprimer un mouvement de rotation aux structures. Ce système laisse évidemment la possibilité d'observer les faits archéologiques sous divers angles de vue, mais autorise surtout la production de profils selon des plans de coupe choisis [ill. 1]. Une capture d'écran au format image permet de vectoriser la section avec un logiciel de dessin. Ce travail, qui n'a pas encore été commencé, demande un temps de formation

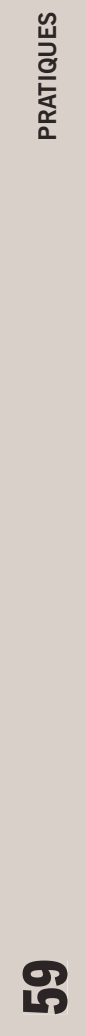

品 
1. Profil restitué de la piscine des thermes d'après la vue en relief. Le nuage de points offre une vision en trois dimensions des faits archéologiques.

Les documents sont exploités

à l'aide d'un logiciel libre

(MeshLab) qui permet

d'imprimer un mouvement

de rotation aux structures.

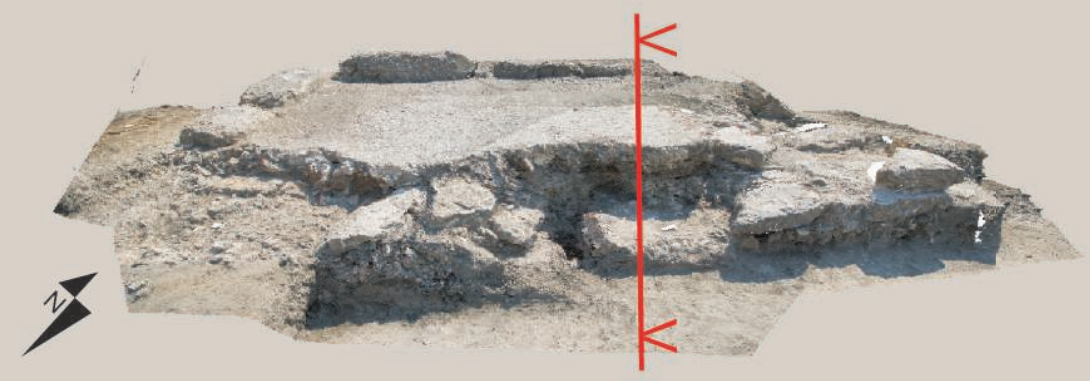

Localisation de la coupe sur la vue 3D

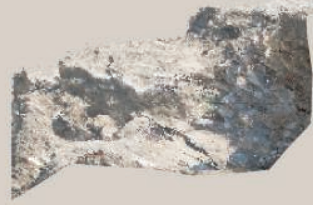

Profil extrait du nuage de points
8

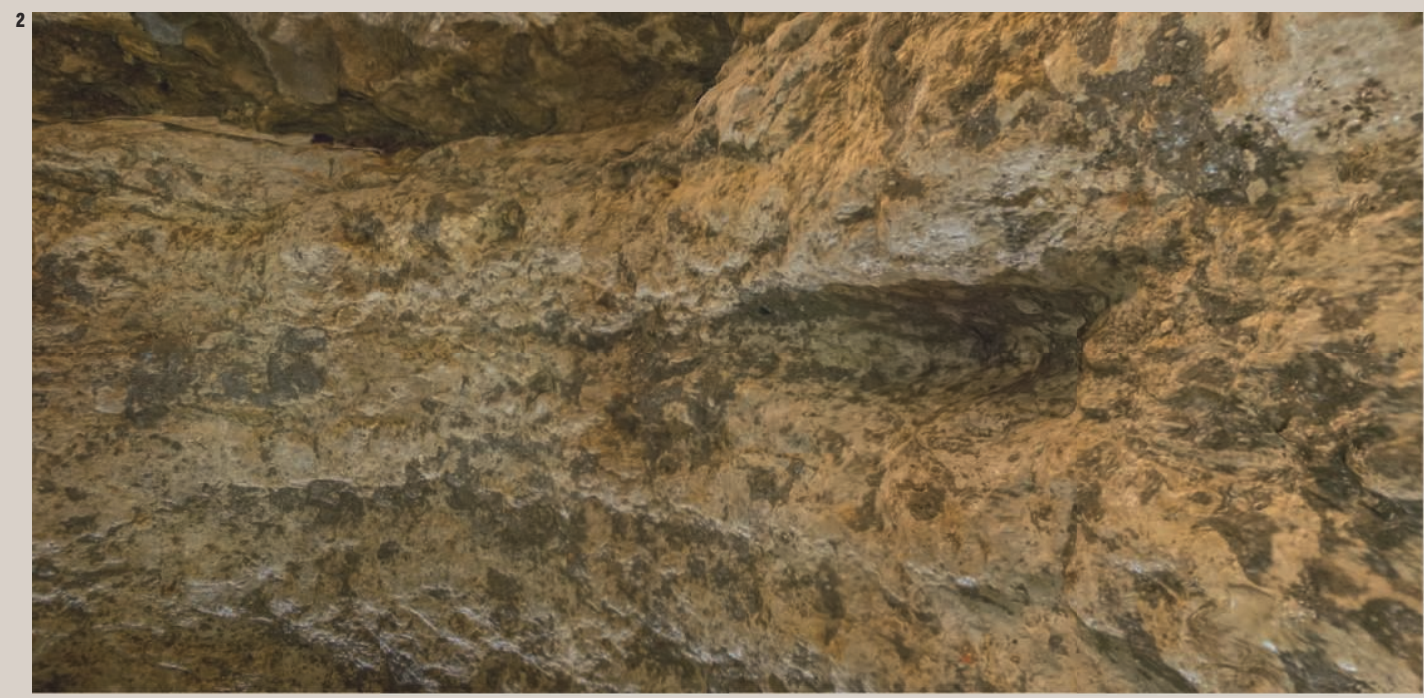

Modèle 3D texturé basé sur le nuage de points

\section{Vue en relief d'une}

des encoches creusées

dans la paroi du puits.

Elles ont été taillées par

les puisatiers gallo-romains

pour permettre l'escalade

des parois.

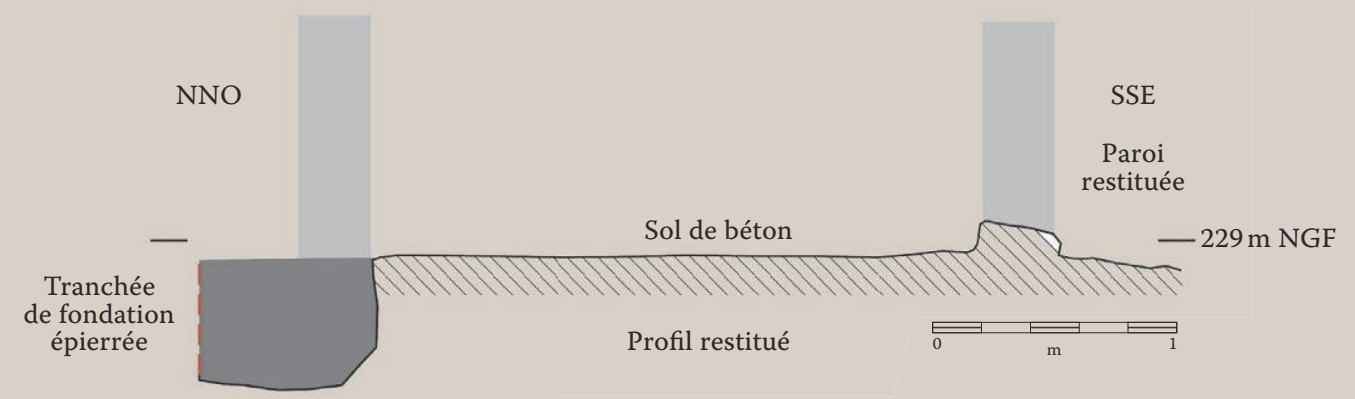


préalable à l'infographiste pour se familiariser avec l'outil. Un autre avantage du nuage de points exploité dans MeshLab est la possibilité d'effectuer des mesures à échelle réelle. Corrélé à la vue en relief, ce logiciel devient un véritable outil d'analyse stratigraphique. Une utilisation qui se vérifie particulièrement bien dans le cas de l'étude du charnier où l'anthropologue peut mesurer précisément les écarts séparant les divers ossements selon un nombre infini de points de vue, la vision en trois dimensions facilitant la détermination des pièces osseuses. La même remarque pourra être faite pour le puits où la numérisation en relief permet de restituer non seulement le parement, mais aussi le modelé de la paroi karstique qui laisse apparaître les traces de piquetage, et notamment une série d'encoches aménagées pour servir de prises d'escalade. La haute résolution des clichés offre l'opportunité d'examiner en détail chaque entaille et d'en mesurer la dimension [ill. 2], ce que les délais contraints de l'opération et l'inconfort de la technique de fouille n'auraient jamais autorisé avec l'emploi d'une autre méthode de relevé.

\section{Considérations sur la méthode}

$\mathrm{Si}$, comme nous l'avons dit, il est encore trop tôt pour dresser un bilan définitif de cette expérience, nous pouvons formuler une série de remarques concernant la méthode : ses atouts, les réserves qu'elle suscite et les ajustements à apporter.

L'avantage principal du procédé est bien entendu la rapidité avec laquelle s'effectue l'acquisition des données : la couverture photographique de l'ensemble d'un site de $1000 \mathrm{~m}^{2}$ peut ainsi se réaliser en quelques minutes au moyen d'un drone ou en environ une heure depuis le sol. La phase terrain étant la plus coûteuse, tous moyens de réduction des délais d'intervention présentent un grand intérêt, sans parler du bénéfice que trouve l'aménageur à voir la durée du chantier écourtée. Toutefois, il ne faut pas que le gain de temps sur le terrain se solde par un rallongement disproportionné de la phase de traitement de la documentation en post-fouille. Même si cette phase avait été achevée pour la fouille d'Entrains, il aurait été difficile de répondre à cette question sur la base d'un exemple précurseur puisque l'exploitation des résultats impose encore une période de formation aux logiciels de traitement 3D. Ce n'est qu'en renouvelant les expériences, une fois qu'archéologues et infographistes seront familiarisés avec les nouveaux outils et qu'un protocole de gestion de la documentation primaire aura été systématisé, qu'un jugement objectif pourra être réellement posé.

Un autre intérêt remarquable est le faible encombrement logistique qu'implique la méthode. À l'exception des cas où un drone est utilisé, la couverture photographique peut être réalisée avec un simple appareil photo reflex paramétré selon les besoins des logiciels de traitement. L'exemple du puits est édifiant à ce propos : l'étroitesse du contexte et les conditions d'accès exigeaient l'usage d'un matériel de petite taille manipulable par la main de l'homme, sans quoi le relevé tridimensionnel n'aurait pas pu se faire, surtout dans des délais aussi courts.

Il convient d'insister également sur le niveau de précision qu'offre cette technique en termes d'acquisition de données, la résolution pouvant être dans certains cas de l'ordre du millimètre. Une qualité qui est bien souvent supérieure au besoin de l'étude archéologique et qui, nous l'avons vu, pose ensuite des problèmes de gestion des fichiers en raison de leur poids électronique trop lourd. Il reste donc à trouver un juste milieu entre la qualité des images fournies et le degré d'exigence souhaité par les archéologues.

Enfin, on ne manquera pas de remarquer que le relevé par photogrammétrie permet de conserver la couleur des structures. Une vision des couleurs qui dépend évidemment des conditions d'observation et de la luminosité ambiante, mais qui rapproche encore davantage la vue en relief de la réalité et facilite ainsi l'identification d'éléments à isoler, comme dans le cas des os du charnier ou des pierres des murs.

\section{Préserver l'objet d'étude avant tout}

Nous ne pouvons conclure sans rappeler un principe général, auquel tout archéologue est particulièrement attaché. C'est qu'importe la méthode de relevé utilisée, l'objet d'étude doit demeurer le fait archéologique et non l'image ou le modèle numérique qui en a été produit. Chacun sait qu'un relevé manuel présente l'intérêt de devoir passer du temps face à la structure, ce qui permet d'être attentif à de nombreux détails et surtout de pouvoir vérifier en direct les problèmes posés par la stratigraphie. Or une accélération de la phase d'acquisition peut avoir pour conséquence une diminution du temps passé sur le terrain et un risque de manquer une information. Aussi à l'exception du puits et du charnier qui sont des cas très particuliers, nous avons privilégié l'utilisation de cette méthode d'enregistrement $3 \mathrm{D}$ pour des structures a priori simples. C'était notamment le cas des murs qui ne présentaient pas de phasages complexes. La démarche peut se révéler optimale si les données brutes sont traitées dans la foulée pour servir de support d'enregistrement, par exemple en annotant directement les images ou bien en délimitant des reprises de maçonnerie ou de grandes séquences stratigraphiques. Encore faut-il pour cela que le volume de données collectées soit adapté aux besoins de l'étude archéologique de manière à ce que le traitement informatique reste rapide et que la documentation puisse être retournée sur le terrain dans des délais relativement brefs.

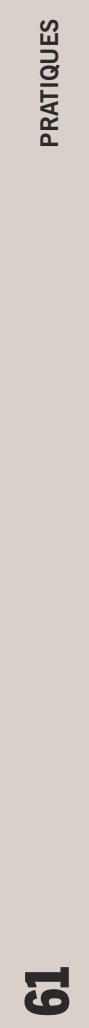

후 